\title{
MIMO in Tunnel: Impact of Polarization and Array Orientation on the Channel Characteristics
}

\author{
F. Challita, P. Laly, M. Lienard, D. P. Gaillot and \\ P. Degauque \\ Lille University/IEMN/TELICE \\ Villeneuve d'Ascq, France \\ Martine.Lienard@univ-lille1.fr
}

\author{
J. M. Molina-Garcia-Pardo \\ Dpto. Tecno. de la Información y las Comunicaciones \\ Universidad Politecnica de Cartagena \\ Cartagena, Spain \\ josemaria.molina@upct.es
}

\author{
W. Joseph \\ Ghent University/iMinds \\ Gent, Belgium \\ wout.joseph@ugent.be
}

\begin{abstract}
Polarimetric characteristics of the propagation channel in a straight tunnel and in a frequency band around $1.3 \mathrm{GHz}$, are deduced from measurements using a real-time MIMO channel sounder. Emphasis is on the cross-polarization discrimination factor and, for a MIMO configuration, on the correlation between transmitting and receiving array elements, depending on the array orientation and on the polarization of the transmitting elements.
\end{abstract}

\section{INTRODUCTION}

Wireless communications in confined areas, as in road and railway tunnels have already been widely studied. Path loss and delay spread have first been determined for a single-input single-output (SISO) link but the new generation of communication systems is based on multiple-input multipleoutput (MIMO) systems. For frequencies on the order or greater than $1 \mathrm{GHz}$, it was shown than the channel capacity can be improved by using MIMO instead of SISO links. However, this improvement is strongly related to MIMO channel characteristics as, for example, the correlation between the transmitting $(\mathrm{Tx}) /$ receiving $(\mathrm{Rx})$ array elements which are usually co-polarized [1]. To decrease this correlation while keeping non prohibitive element spacing, a possible solution could be to combine MIMO and polarization diversity. Determination of the statistical polarimetric channel properties and the wideband channel characteristics, are of prime importance.

Such MIMO measurements are often made by using a vector network analyzer or an equivalent system, a cable or a fiber optics thus connecting $\mathrm{Tx}$ and $\mathrm{Rx}$ as in [2]. The main drawbacks are firstly that the number of measurement points along the tunnel is quite limited and, secondly, that the tunnel must be closed to traffic. In this paper measurements have thus been carried out with a real time channel sounder, the receiving part being onboard a vehicle. Different orientations of the antenna arrays together with various polarizations of the array elements have been tested to show their influence on the mean path loss, on the cross polar (X-polar) discrimination factor
(XPD) and on the correlation between $\mathrm{Tx}$ or $\mathrm{Rx}$ elements. Section II briefly recalls the main features of the sounder that we have designed, while Section III describes the geometry of the tunnel and the different configurations of the $\mathrm{Tx} / \mathrm{Rx}$ antenna arrays. Section IV presents some results on the influence of polarization on channel characteristics.

\section{MaIn Features of THE ChanNEL SOUNDER}

The MIMO $(8,16)$ sounder has a center frequency of $1.35 \mathrm{GHz}$ with a transmission bandwidth of $80 \mathrm{MHz}$. It is based on an interleaved frequency division multiplexing technique. Since the size Ntx of the Tx array can be chosen, the 6560 subcarriers can be allocated either to only one antenna or distributed among all Tx antennas. As an example for $N t x=8$, the frequency spacing between subcarriers transmitted by each antenna is $97.66 \mathrm{kHz}$. In the time domain, the maximum duration of the channel impulse response is $10.24 \mu$ s with a time resolution of $12.5 \mathrm{~ns}$.

A switch with $50 \mathrm{~dB}$ isolation is used to change the polarization of each dual-polarized $\mathrm{Tx}$ antenna to avoid doubling the RF front end. Therefore, 2 OFDM symbols are successively sent, the first one for one polarization and the other one for the orthogonal polarization. On the contrary, at $\mathrm{Rx}$, the signals measured at each of the 2 outputs of dualpolarized patch antennas are simultaneously stored. With symbol duration of about $82 \mu \mathrm{s}$, the acquisition time of a full polarimetric channel matrix (deduced from the reception of 2 successive symbols) is about $300 \mu \mathrm{s}$.

\section{GeOMETRICAL CONFIGURATION}

Measurements have been carried out in a 2-way road tunnel but one way was closed to traffic. The configuration of the tunnel and the implementation of the Tx and Rx arrays are shown in Fig. 1. The tunnel, $600 \mathrm{~m}$ long, has a maximum width of $9.7 \mathrm{~m}$ and a maximum height of $4.6 \mathrm{~m}$. Each antenna of the 8 -element uniform arrays (numbered 1 to 8 in Fig. 1) is a dualpolarized patch antenna, the port isolation and cross 
polarization being larger than $30 \mathrm{~dB}$ and $25 \mathrm{~dB}$ across the whole frequency band, respectively.

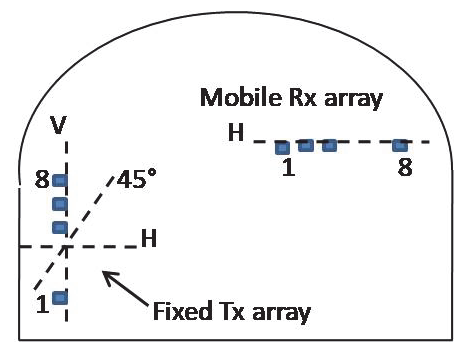

Fig. 1. Cross section of the tunnel and positions of the Tx and Rx arrays.

The spacing between the centers of each successive Tx/Rx element is $17 \mathrm{~cm}$, i.e. 0.76 wavelength $(\lambda)$ at $1.35 \mathrm{GHz}$. The orientation of each path in the cross section of the tunnel can be changed and, in the experiments, the dual polarizations of each patch are either vertical (V) / horizontal $(\mathrm{H})$ or $+45^{\circ} / 45^{\circ}$. Successive orientations of the Tx array were also considered: $\mathrm{H}, \mathrm{V}$ or $45^{\circ}$ as shown in Fig. 1 . In any case, the Rx array is horizontal and put at the rear of a small van, at a height of about $2 \mathrm{~m}$ above ground

\section{EXPERIMENTAL RESULTS}

In a first step, some results on the polarization effect on path loss are presented, including statistical values of the discrimination factor. Then the correlation between array elements are studied as a function of their polarization.

\section{A. Field Variation and Cross Polar Discrimination Factor}

As an example, curves in Fig. 2 show the variation of the channel gain $\mathrm{G}$ between antennas Tx1 and Rx1, for co-polar (VV) or cross-polar (VH) polarizations, the first and second letters referring to the polarization of $\mathrm{Tx}$ and of $\mathrm{Rx}$, respectively. $G$ has been averaged over the whole transmitting bandwidth to improve the clarity of the Figure by avoiding strong fluctuations of the curves when deep fading occur in this frequency selective channel. Fig. 2 shows that the average additional attenuation for X-polar does not depend on the distance $d$ between Tx and Rx.

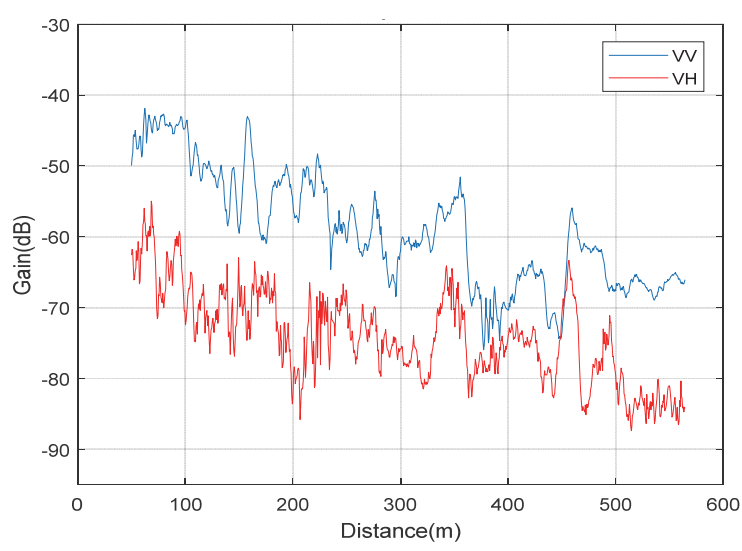

Fig. 2. Channel gain for co- and cross- polarization
The cumulative distribution function (cdf) of $\mathrm{G}$ related to the previous configuration is plotted in Fig. 3. We see that curves related to $\mathrm{VV}$ and $\mathrm{VH}$ are parallel and that XPD is about $14 \mathrm{~dB}$. The same approach has been made for the various antennas and polarizations mentioned in Section III in order to deduce statistical results.

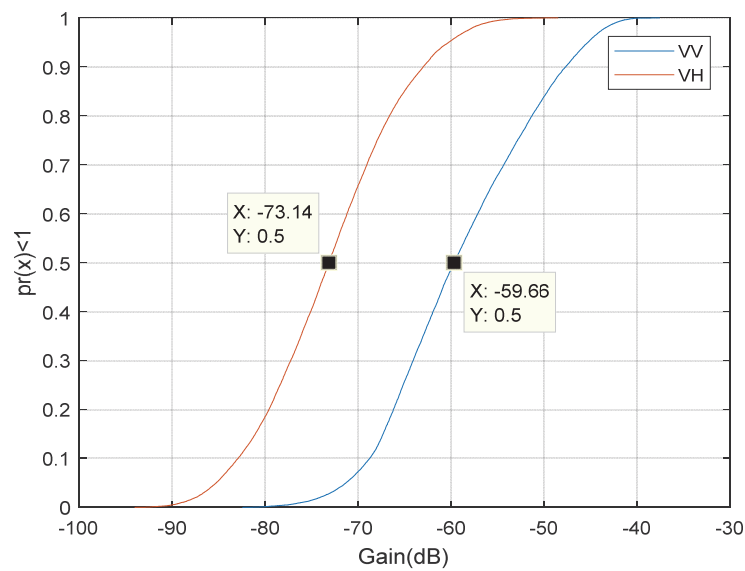

Fig. 3. Cdf of the gain for co- and X-polarizations

\section{B. Correlation between Array Elements}

For co-polarized $\mathrm{Tx}$ or $\mathrm{Rx}$ elements, the correlation increases with the distance Tx-Rx. For example, for an element spacing of $34 \mathrm{~cm}(1.5 \lambda)$, the correlation varies from 0.7 to 0.9 when $d$ increases from $50 \mathrm{~m}$ to $300 \mathrm{~m}$. To decrease correlation while minimizing the spacing between array elements, a solution could be using Rx and/or Tx polarization diversity, the elements of the linear array being $\mathrm{H}$ and $\mathrm{V}$ successively polarized. However, X-polar reception leads to a strong decrease of the Rx power as shown previously. To cope with this problem, a possible solution could be to use Tx antennas having a polarization of $45^{\circ}$ and to receive the $\mathrm{H}$ component on an $\mathrm{Rx}$ element and the $\mathrm{V}$ component on an adjacent element.

For the same spacing of Rx elements as previously, this leads to an average correlation of 0.6 at $300 \mathrm{~m}$ instead of 0.9 when using successive co-polar elements. A statistical approach has been made by considering all $\mathrm{Tx}$ and $\mathrm{Rx}$ elements. Lastly, the increase in MIMO ergodic capacity by introducing polarization diversity was also studied.

\section{ACKNOWLEDGEMENT}

This work has been done in the frame of the ELSAT2020 project co-financed by the European Union with the European Regional Development Fund, the French state and the Hauts de France Region Council.

\section{REFERENCES}

[1] P. Laly, M. Lienard, P. Degauque, C. Sanchis-Borras, and J. M. Molina Garcia Pardo, "Propagation in road tunnels: Predicted performances of LTE precoding techniques," IEEE RADIO Conf., Proc. pp. 1-1, 25 - 28 Sept. 2017

[2] R. Sun, D. W. Matolak, C. Tao, L. Liu, Z. Tan dna T. Zhou, "Investigation of MIMO channel characteristics in a two-way section tunnel at $1.4725 \mathrm{GHz}$," Hindawi Int. J. of Antennas and Propag., Article ID 3693149, 12 pages, 2017 